\section{The incidence and the risk factors for iatrogenic retinal breaks during pars plana vitrectomy}

\begin{abstract}
Purpose To establish the frequency and the risk factors for iatrogenic retinal breaks during three-port pars plana vitrectomy (PPV).

Methods A total of 2471 PPV operations were included in the study. The study period was between 2001 and 2010, all the data were entered in an electronic patient record database. All 270 consecutive eyes of 270 patients developing iatrogenic retinal breaks during primary PPV were matched to 270 controls. Univariate and multivariate analysis were performed to establish the risk factors. Results The median age of the patients with iatrogenic breaks was 60.06 years; male to female ratio was $140 / 130$. The overall frequency of iatrogenic retinal breaks was $10.09 \%$. The frequency of iatrogenic retinal breaks for eyes undergoing PPV for tractional retinal detachment (TRD) was $32.45 \%$. The lens status was phakic in $79.6 \%$ of the eyes, with iatrogenic breaks compared with $34.4 \%$ of the eyes in control group $(P<0.001)$. Posterior vitreous was attached in $58.9 \%$ of the eyes with iatrogenic breaks compared with $50.4 \%$ of the eyes in control group $(P=0.04)$. Internal

limiting, epiretinal, proliferative, or

fibrovascular membrane removing
\end{abstract}

St Thomas'Hopital, London, UK

Correspondence:

M Dogramaci,

St Thomas'Hopital, Westminster Bridge Road, London SE17EH, UK

Tel: + $44(0) 7751933917$;

Fax: + 44 (0)2071889193

E-mail: mahmutdogramaci@ hotmail.com

Received: 6 September 2011

Accepted in revised form:

11 January 2012

Published online:

17 February 2012 manoeuvers were performed in $71.1 \%$ of the eyes with iatrogenic breaks compared with $61.9 \%$ of the eyes in control group $(P=0.052)$. Conclusion Eyes undergoing PPV for TRD had significantly higher frequency of iatrogenic retinal breaks compared with other subgroups $(P=0.0001)$. Phakia and absence of PVD were found to be risk factors for iatrogenic retinal breaks.

Eye (2012) 26, 718-722; doi:10.1038/eye.2012.18; published online 17 February 2012

Keywords: pars plana vitrectomy; iatrogenic retinal breaks; retinal detachment
M Dogramaci, EJK Lee and TH Williamson

Introduction

Modern plana vitrectomy (PPV) operations have increasing numbers of indications. Rhegmatogenous retinal detachment, macular hole, epiretinal membrane (ERM), vitreomacular traction syndrome, proliferative diabetic retinopathy, and retained lens matter after complicated cataract surgery are the main indications.

Secondary retinal detachment caused by intraoperative iatrogenic retinal breaks remains the most common sight-threatening complication of this surgery despite the recent advances in wide-angle viewing systems and instrumentation. ${ }^{1-4}$ The frequency of iatrogenic retinal breaks during PPV has been reported to range from 0 to $24 \%, 3,5-13$ with post PPV retinal detachment occurring in $0-15.8 \% .^{3,7-17}$ Unlike previous studies, in this study, the data are prospectively collected and the cases were matched to controls without iatrogenic breaks to establish the frequency, and risk factors for intraoperative iatrogenic retinal breaks in the largest reported series of patients undergoing PPV.

Materials and methods

All primary PPV procedures, performed or supervised by one author (THW), were entered in an electronic patient record database (available with Vitreoretinal Surgery book published by Springer). ${ }^{18}$ In this database, patients' demographics, primary indications, preoperative findings, and the intraoperative and postoperative complications were prospectively recorded. Eyes developing iatrogenic retinal breaks during PPV operations performed between January 2001 and December 2010 were matched to eyes with no iatrogenic retina breaks in the same database for age, sex, and primary indication for surgery. 
Before surgery, each case underwent $360^{\circ}$ fundal examination at the slit lamp, although this was limited in eyes with dense media opacity (for example, vitreous hemorrhage). All patients subsequently underwent standard 20 or 23-gauge PPV using Alcon Accurus (Alcon Laboratories, Fort Worth, TX, USA) vitrectomy systems and the Binocular Indirect Ophthalmo Microscope (BIOM, Oculus Inc., Wetzlar, Germany) non-contact, wide-angle viewing system. The operations were performed under either general anaesthesia or local anaesthetics by subtenons and/or peribulbar injection. Sclerotomies were placed $3.5 \mathrm{~mm}$ from the limbus in pseudophakic or aphakic eyes and $4 \mathrm{~mm}$ from the limbus in phakic eyes. Cataract operation was performed at commencement of the procedure when a combined procedure was planned, so eyes that underwent combined cataract operation and PPV were not considered as phakic. Sclerotomy cannulae were used for 23 gauge PPVs. All vitrectomy cutters were single-use disposable instruments, and were not reused in accordance with local infection control protocols and manufacturers' recommendations. Posterior hyaloid was surgically separated in all cases with no apparent preoperative posterior vitreous detachment (PVD). The separation was initiated by active aspiration (maximum vacuum $250-600 \mathrm{~mm} \mathrm{Hg}$ ) over the optic disc using vitrectomy probe in cutter-off mode. A contact lens was used as necessary for part of the procedure, for example, during membrane peel or delamination. As per the departmental policy, internal search was performed in all cases undergoing PPV, using scleral indentation with endoillumination and the wide-angle BIOM viewing system at the conclusion of the surgery. Identified breaks were treated with cryotherapy or laser retinopexy, followed by intraocular gas or oil tamponade. All cases were performed by an experienced vitreoretinal surgeon, at the level of consultant or fellow-in-training.

Iatrogenic retinal break was defined as a full thickness break in the neurosensory retina created during surgery. This included retinal tears at the posterior edge of vitreous base and breaks posterior to the equator. Preoperatively identified breaks were not counted, and preexisting retinal detachment cases were excluded from the study.

Approval was obtained from the institutional research ethics committee of Guy's \& St Thomas' Hospitals NHS Foundation Trust, and the study conformed to the Declaration of Helsinki. Statistical analyses were performed using paired $t$ test, Wilcoxon test, MacNemar test, Fisher's exact test, and multiple logistic regression test as required, using SPSS software version 17.0 (SPSS Inc., Chicago, IL, USA).

\section{Results}

A total of 2472 primary PPV operations, eligible for inclusion in the study for the period between 2001 and 2010 , were analysed. Intraoperative iatrogenic retinal breaks developed in 270 out of a total of $2472(10.09 \%)$ eyes included in the study, 98 out of $302(32.45 \%)$ eyes with tractional retinal detachment (TRD), 66 out of 409 $(16.13 \%)$ eyes with macular hole, 26 out of $277(9.38 \%)$ eyes with macular pucker, 42 out of $512(8.06 \%)$ eyes with vitreous haemorrhage (VH), 10 out of $146(6.84 \%)$ eyes with ocular trauma, 11 out of $248(4.43 \%)$ eyes with vitritis, 4 out of 141 (2.83\%) eyes with dropped nucleus, and finally 13 out of 427 (3.04\%) eyes with other diagnoses. Other diagnoses comprised choroidal biopsy, myopic macular schisis, and optic disc pit maculopathy, suprachoroidal haemorrhage, choroidal neovascular membrane, macular oedema, asteroid hyalosis, vitreomacular traction, and radial optic neurotomy for central retinal vein occlusion. Eyes with TRD and macular holes had a significantly higher frequency of intraoperative iatrogenic breaks compared with the sum of the other subgroups $(P<0.0001)$. Table 1 summarises the frequency of iatrogenic breaks in each subgroup.

The 270 eyes with iatrogenic breaks were matched to 270 controls for their primary indication for surgery, sex, and age. The primary indication for PPV in 98 eyes was $\mathrm{TRD}$, in 66 eyes was macular hole, in 42 eyes was VH, in 26 eyes was macular pucker, in 13 eyes was other diagnoses, in 10 eyes was trauma, and finally in 4 eyes was dropped nucleus within the iatrogenic break group and the control group. Male to female ratio was 140 to 130 in the iatrogenic break group and the control group.

Table 1 Summarises the frequency of iatrogenic breaks in each subgroup

\begin{tabular}{lccc}
\hline $\begin{array}{l}\text { Indication } \\
\text { for vitrectomy }\end{array}$ & $\begin{array}{c}\text { Number of } \\
\text { the eyes with } \\
\text { iatrogenic breaks }\end{array}$ & $\begin{array}{c}\text { Total } \\
\text { number of } \\
\text { the eyes }\end{array}$ & $\begin{array}{c}\text { \% of eyes } \\
\text { with iatrogenic } \\
\text { breaks within } \\
\text { the subgroup }\end{array}$ \\
\hline TRD & 98 & 302 & $32.45 \%$ \\
Macular hole & 66 & 409 & $16.13 \%$ \\
Macular pucker & 26 & 277 & $9.38 \%$ \\
VH & 42 & 521 & $8.06 \%$ \\
Trauma & 10 & 146 & $6.84 \%$ \\
Vitritis & 11 & 248 & $4.43 \%$ \\
Others & & 427 & $3.04 \%$ \\
Dropped nucleus & 13 & 141 & $2.83 \%$ \\
Total & 4 & 2471 & $10.09 \%$ \\
\hline
\end{tabular}

Abbreviations: TRD, tractional retinal detachment; $\mathrm{VH}$, vitreous haemorrhage.

${ }^{a}$ These comprised choroidal biopsy, myopic macular schisis, and optic disc pit maculopathy, suprachoroidal hemorrhage, choroidal neovascular membrane, macular oedema, asteroid hyalosis, vitreomacular traction, and radial optic neurotomy for central retinal vein occlusion. 
The median age was 60.06 years for the iatrogenic break group and 60.40 years in the control group.

The initial mean best-corrected visual acuity (BCVA) was LogMAR 1.33 (counting fingers $(\mathrm{CF})$ ) in the iatrogenic break group compared with LogMAR 1.27 (CF) in the control group. The final mean BCVA was LogMAR $0.47(20 / 50)$ in the iatrogenic break group and LogMAR $0.38(20 / 40)$ in the control group. There was no significant difference between the groups in the mean initial and the mean final BCVA $(P=0.493$ and 0.167$)$. The mean pre and post operation difference in the BCVA was 0.85 LogMAR units in the iatrogenic break group and 0.90 LogMAR units in the control group, which was not statistically significant $(P=0.586)$.

The operations were performed on the right side in 136 out of $270(50.3 \%)$ eyes with iatrogenic breaks compared with 135 (50\%) eyes in the control group $(P=0.682)$. General anaesthetics were given to 205 out of $270(75.9 \%)$ eyes with iatrogenic breaks compared with 212 out of $270(78.5 \%)$ eyes in the control group $(P=0.28)$. A 23-gauge PPV was used in 12 out of $270(4.5 \%)$ eyes with iatrogenic break compared with $3(1.2 \%)$ eyes in the control group; the difference was not statistically significant.

The lens status was phakic in 215 out of $270(79.6 \%)$ eyes with iatrogenic breaks compared with 93 out of 270 $(34.4 \%)$ eyes in the control group; this was statistically significant $(P<0.001)$. PVD was absent in 159 out of 270 (58.9\%) eyes with iatrogenic breaks compared with 136 out of $270(50.4 \%)$ eyes in the control group. The difference in the prevalence of PVD between the cases and the controls was statistically significant $(P=0.04)$ odds ratio $=1.589,95 \%$ CI (1.020-2.475).

Peeling internal-limiting membrane (ILM), ERM, fibrovascular membranes, or proliferative membranes were performed in 192 out of 270 (71.1\%) eyes with iatrogenic breaks compared with 167 out of 270 (61.9\%) eyes in the control group; this difference was statistically significant on univariate analysis $(P=0.01)$ and marginally significant on multivariate analysis $(P=0.052)$. As the cases and the controls were collected over the same period, surgical techniques were similar between both groups. Post operative secondary retinal detachment occurred in 13 out of 270 (4.8\%) eyes with iatrogenic retinal breaks compared with 6 out of 270 $(2.2 \%)$ eyes in the control group; this difference was statistically significant on univariate analysis $(P=0.029)$ and not significant on multivariate analysis $(P=0.667)$. The mean onset of post operative RD was 49 days in iatrogenic break group compared with 128 in the control group $(P=0.311)$. The mean postoperative follow up was 1.51 years for the iatrogenic break group compared with 1.33 years for the control group $(P=0.426)$. Table 2 summarises patient demographics, laterality, and preoperative ocular and general status of the eyes in the iatrogenic break group compared with control group.

\section{Discussion}

Intraoperative iatrogenic retinal breaks leading to secondary postoperative retinal detachments are important sight-threatening complication of

Table 2 Summarises patient demographics, laterality, and preoperative ocular and general status of the eyes in the iatrogenic break group compared with control group

\begin{tabular}{|c|c|c|c|c|}
\hline & $\begin{array}{l}\text { Iatrogenic } \\
\text { beak group }\end{array}$ & $\begin{array}{l}\text { Control group, } \\
\text { excluded }\end{array}$ & $\begin{array}{l}\text { Univariate analysis, P-values, } \\
\text { and confidence intervals }\end{array}$ & $\begin{array}{l}\text { Multivariate analysis, } \mathrm{P}-\mathrm{values}, \\
\text { and confidence intervals }\end{array}$ \\
\hline Median age in years (SD) & $60.06(16.26)$ & $60.40(31)^{\mathrm{a}}$ & & \\
\hline Male/female & $140 / 130$ & $140 / 130$ & & \\
\hline Right/left eye ratio & $136 / 134$ & $135 / 135$ & $P=1.00$ & \\
\hline General/local anaesthetics & $205 / 65$ & $212 / 58$ & $P=0.534$ & \\
\hline Initial mean BCVA LogMAR & $1.33(\mathrm{CF})$ & $1.27(\mathrm{CF})$ & $P=0.323$ & \\
\hline Final mean BCVA LogMAR & $0.47(20 / 50)$ & $0.38(20 / 40)$ & $P=0.073$ & $P=0.167$ \\
\hline Mean vision improvement LogMAR & 0.85 & 0.90 & $P=0.452$ & \\
\hline Phakic eyes & $215(79.6 \%)$ & $93(34.4 \%)$ & $\begin{array}{l}P<0.001, \text { odds ratio }=1.701, \\
95 \% \text { CI: } 0.873-3.315\end{array}$ & $\begin{array}{l}P<0.001, \text { odds ratio }=0.102 \\
95 \% \text { CI: } 0.66-0.158\end{array}$ \\
\hline Absence of PVD & $159(58.9 \%)$ & $136(50.4 \%)$ & $\begin{array}{l}P=0.009, \text { odds ratio }=8.638 \\
95 \% \text { CI: } 4.905-15.212\end{array}$ & $\begin{array}{l}P=0.04 \text { Odds ratio }=1.589 \\
95 \% \text { CI: } 1.020-2.475\end{array}$ \\
\hline Membrane removing manoeuvers & $192(71.1 \%)$ & $167(61.9 \%)$ & $\begin{array}{l}P=0.01, \text { Odds ratio: } 3.682, \\
95 \% \text { CI: } 2.124-6.382\end{array}$ & $\begin{array}{l}P=0.052, \text { Odds ratio: } 1.506 \\
95 \% \text { CI: } 0.997-2.276\end{array}$ \\
\hline Post op RDs & $13(4.8 \%)$ & $6(2.2 \%)$ & $\begin{array}{l}P=0.029, \text { Odds ratio: } 11.5 \\
95 \% \text { CI: } 1.898-69.677\end{array}$ & $P=0.667$ \\
\hline Onset of RD mean days (SD) & $49(26.180)$ & $128(152.820)$ & $P=0.435$ & \\
\hline Mean follow up in years & 1.51 & 1.33 & $P=0.249$ & $P=0.426$ \\
\hline 23 gauge & $12(4.5 \%)$ & $3(1.2 \%)$ & $P=1.00$ & \\
\hline
\end{tabular}

Abbreviations: BCVA, best corrected-visual acuity; PVD, posterior vitreous detachment; RD, retinal detachment.

${ }^{a}$ Mean age of the entire group with no iatrogenic break was 61.42 years $(\mathrm{SD}=15)$. 
pars PPV. ${ }^{3,6,7,9,13-17}$ In this case-controlled study, we report the frequency and the risk factors for iatrogenic retinal breaks in the largest published series for eyes undergoing PPV for various indications.

The overall frequency of intraoperative iatrogenic retinal breaks in this study was $10 \%$. This compares with 3 and 15\% reported in other studies. ${ }^{19,20}$ Variation between the studies is likely to be due to the differences in inclusion criteria and case profile. We included both anterior and posterior iatrogenic retinal breaks observed during PPV operation in our study. Literature review shows that some studies, in line with ours, included both anterior and posterior iatrogenic retinal breaks. ${ }^{3,6,10}$ Other studies excluded posterior iatrogenic retinal breaks, ${ }^{11,12,19,20}$ and one study did not specify. ${ }^{8}$

The frequency of the intraoperative iatrogenic retinal breaks differs according to the variation in the case profile. ${ }^{20}$ Our study showed that eyes presenting with TRD have the highest incidence of $32.45 \%$ of intraoperative iatrogenic retinal breaks compared with other subgroups. Similarly, Yashin et $a l^{20}$ found a higher incidence of iatrogenic breaks in the TRD subgroup in their series. Likewise, we found that eyes presenting with macular holes have a frequency of $16.13 \%$ of intraoperative iatrogenic retinal breaks compared with 0 and $18 \%$ incidence rate reported in previous studies. ${ }^{6,7,9,13,20}$ In contrast, we found that eyes presenting with macular pucker have a frequency of $9.38 \%$ of intraoperative iatrogenic retinal breaks, which is higher than $0.6-6.9 \%$ incidence rate reported in previous retrospective studies. This is likely to be because of the fact that our data was collected prospectively.

Interestingly, we found a higher rate of $4.8 \%$ postoperative retinal detachment among the eyes with identified intraoperative iatrogenic retinal breaks compared with $2.2 \%$ rate among eyes within the control group; the difference was statistically significant $(P=0.029)$ on univariate analysis but not on multivariate analysis $(P=0.311)$. Similar differences were noticed in the rate of postoperative retinal detachment in other studies. ${ }^{1,20}$ It has previously been found that peripheral retinal breaks identified and treated at the time of primary PPV, do not correspond to the causative break(s) for subsequent retinal detachment. ${ }^{20}$ This suggests that a higher level of care may need to be taken during the internal search in eyes with identified iatrogenic breaks in order to rule out multiple breaks.

In addition to the variation of frequency of iatrogenic retinal breaks according to the primary indication for surgery, we found a significant association between phakia and the frequency of intraoperative iatrogenic breaks $(P<0.001)$. This concurs with the findings in other studies. ${ }^{8,10,20}$ It may be because of the higher risk of vitreous base traction when sclerotomies are positioned more posteriorly in phakic eyes. Alternatively, pseudophakic/aphakic eyes may permit more comprehensive peripheral vitrectomy, with fewer instrument changes, thereby reducing the incidence of entry site complications. However, Chung et al ${ }^{6}$ and Wimpissinger et $a l^{12}$ did not find correlation between retinal break formation and lens status. ${ }^{6,12}$

Absence of preoperative PVD was significantly associated with a higher frequency of iatrogenic retinal breaks in our study $(P=0.04)$. This agrees with the findings of previous studies, where they found a strong association between surgical induction of PVD and the frequency of iatrogenic breaks in eyes undergoing PPV for various indication excluding RRD. ${ }^{6,20}$

The practice of peeling ILM, ERM, fibrovascular membranes, or proliferative membranes during PPV was another risk factor that was associated with higher frequency of iatrogenic retinal breaks in this study. This association was statistically more significant on univariate analysis and marginally significant on multivariate analysis ( $P=0.01$ and 0.052 , respectively). It has been noted in previous studies that using intraocular scissors and/or forceps for membrane peeling and cutting during PPV increases the risk of iatrogenic retinal breaks. ${ }^{19}$ It is conceivable that such instruments result in an increased vitreous drag with associated retinal breaks. The follow-up duration in the iatrogenic break group was longer than the control group, but this was not significant.

A 23-guage PPV was significantly associated with a higher frequency of iatrogenic retinal breaks. However, it is difficult to comment on this finding because of the limited numbers of 23 gauges PPVs during the study period. This study did not look into the location of the iatrogenic breaks, axial length, or the severity of myopia of the eyes involved.

In conclusion, iatrogenic retinal breaks are more common in TRD and macular hole subgroups, and in phakic eyes and in eyes with no PVD. ILM, ERM, fibrovascular membrane, or proliferative membrane peeling during PPV is marginally associated with a higher frequency of iatrogenic breaks.

\section{Summary}

\section{What was known before}

- Secondary retinal detachment caused by intraoperative iatrogenic retinal breaks remains the most common sight-threatening complication of this surgery, despite the recent advances in wide-angle viewing systems and instrumentation.

What this study adds

- This case-control study establishes the frequency and risk factors for intraoperative iatrogenic retinal breaks in the largest reported series of patients undergoing PPV. 


\section{Conflict of interest}

The authors declare no conflict of interest.

\section{References}

1 Moore JK, Scott IU, Flynn Jr HW, Smiddy WE, Murray TG, Kim JE et al. Retinal detachment in eyes undergoing pars plana vitrectomy for removal of retained lens fragments. Ophthalmology 2003; 110: 709-713.

2 Heier JS, Topping TM, Frederick Jr AR, Morley MG, Millay $\mathrm{R}$, Pesavento RD. Visual and surgical outcomes of retinal detachment following macular hole repair. Retina 1999; 19: 110-115.

3 de Bustros S, Thompson JT, Michels RG, Rice TA, Glaser $\mathrm{BM}$. Vitrectomy for idiopathic epiretinal membranes causing macular pucker. Br J Ophthalmol 1988; 72: 692-695.

4 Oyakawa RT, Schachat AP, Michels RG, Rice TA. Complications of vitreous surgery for diabetic retinopathy. I. Intraoperative complications. Ophthalmology 1983; 90: 517-521.

5 Carter JB, Michels RG, Glaser BM, De Bustros S. Iatrogenic retinal breaks complicating pars plana vitrectomy. Ophthalmology 1990; 97: 848-853.

6 Chung SE, Kim KH, Kang SW. Retinal breaks associated with the induction of posterior vitreous detachment. Am J Ophthalmol 2009; 147: 1012-1016.

7 Krohn J. Duration of face-down positioning after macular hole surgery: a comparison between 1 week and 3 days. Acta Ophthalmol Scand 2005; 83: 289-292.

8 Moore JK, Kitchens JW, Smiddy WE, Mavrofrides EC, Gregorio G. Retinal breaks observed during pars plana vitrectomy. Am J Ophthalmol 2007; 144: 32-36.

9 Sjaarda RN, Glaser BM, Thompson JT, Murphy RP, Hanham A. Distribution of iatrogenic retinal breaks in macular hole surgery. Ophthalmology 1995; 102: 1387-1392.
10 Tan HS, Mura M, de Smet MD. Iatrogenic retinal breaks in 25-gauge macular surgery. Am J Ophthalmol 2009; 148: 427-430.

11 Theocharis IP, Alexandridou A, Gili NJ, Tomic Z. Combined phacoemulsification and pars plana vitrectomy for macular hole treatment. Acta Ophthalmol Scand 2005; 83: 172-175.

12 Wimpissinger B, Binder S. Entry-site-related retinal detachment after pars plana vitrectomy. Acta Ophthalmol Scand 2007; 85: 782-785.

13 Guillaubey A, Malvitte L, Lafontaine PO, Hubert I, Bron A, Berrod JP et al. Incidence of retinal detachment after macular surgery: a retrospective study of 634 cases. Br J Ophthalmol 2007; 91: 1327-1330.

14 Michels RG. Vitrectomy for macular pucker. Ophthalmology 1984; 91: 1384-1388.

15 Banker AS, Freeman WR, Kim JW, Munguia D, Azen SP. Vitrectomy for Macular Hole Study Group. Vision-threatening complications of surgery for fullthickness macular holes. Ophthalmology 1997; 104: 1442-1452.

16 Park SS, Marcus DM, Duker JS, Pesavento RD, Topping TM, Frederick Jr AR et al. Posterior segment complications after vitrectomy for macular hole. Ophthalmology 1995; 102: $775-781$.

17 Tabandeh H, Chaudhry NA, Smiddy WE. Retinal detachment associated with macular hole surgery: characteristics, mechanism, and outcomes. Retina 1999; 19: 281-286.

18 Williamson T. Vitreoretinal Surgery. Springer: Berlin, 2008 ISBN:3540375813.

19 Al-Harthi E, Abboud EB, Al-Dhibi H, Dhindsa H. Incidence of sclerotomy-related retinal breaks. Retina 2005; 25: 281-284.

20 Ramkissoon Y, Shaheen S, Shah P, Wong S, Sullivan P. Risk of iatrogenic peripheral retinal breaks in 20-G pars plana vitrectomy. Ophthalmology 2010; 117: 1825-1830. 